\title{
Prevalencia de Caries Temprana de la Infancia y sus Factores Asociados en Niños Chilenos de 2 y 4 Años
}

\author{
Prevalence of Early Childhood Caries and Associated \\ Factors in 2 and 4 Year-Old Chilean Children
}

\author{
Carlos Zaror Sánchez, ;*; Patricia Pineda Toledo* \& Juan José Orellana Cáceres ${ }^{* * *}$
}

ZAROR, S. C.; PINEDA, T. P. \& ORELLANA, C. J. J. Prevalencia de caries temprana de la infancia y sus factores asociados en niños chilenos de 2 y 4 años. Int. J. Odontostomat., 5(2):171-177, 2011.

RESUMEN: Se realizó un estudio de corte transversal durante el año 2008 con una muestra 301 niños de 2 y 4 años que asisten por primera vez al servicio dental del Hospital de Calbuco, cuyo objetivo fue determinar la prevalencia de caries temprana de la infancia (CTI), severa caries temprana de la infancia (CTI-S) y sus factores asociados. El diagnóstico de caries siguió los criterios establecidos por la OMS, considerando a las caries incipientes dentro del componente caries. Además se investigó su relación con factores socio-demográficos, dietarios y hábitos de higiene oral. La información fue analizada usando test exacto de Fisher y modelos de regresión logística. La prevalencia de CTI fue de un $70 \%$ con una severidad de un $52 \%$. Se encontró asociación estadísticamente significativa entre CTI y variables como edad, ruralidad, estado nutricional, uso de biberón, uso biberón nocturno e índice de higiene oral simplificado $(p<0,05)$. Del estudio multivariado se determinó que a los 2 años el usar biberón nocturno es un factor protector $(\mathrm{OR}=2,6)$. La alta prevalencia de CTI en la población estudiada evidencia la necesidad de incorporar programas educativos y preventivos durante el primer año de vida.

PALABRAS CLAVE: Caries temprana de la infancia, prevalencia, epidemiología, factores de riesgo.

\section{INTRODUCCIÓN}

La Caries Temprana de la Infancia (CTI) es una forma severa y particular de caries, de carácter multifactorial, que afecta la dentición temporal de lactantes y niños pre-escolares comprometiendo por lo general numerosos dientes, y produciendo una rápida destrucción e infección subsiguiente del tejido dentario (Horowitz, 1998).

Si bien la etiología de a CTI es multifactorial se ha establecido que el streptococcus mutans (S.M), inmerso en un biofilm bacteriano, es su principal agente causal, efecto potenciado por características en la dieta de los pacientes infantiles. Los hidratos de carbono fermentables presentes en la dieta son metabolizados por las bacterias produciendo una alteración en la homeostasis bacteriana al disminuir el $\mathrm{pH}$ mediante la producción de ácidos y la consecuente desmine- ralización de los tejidos dentales.(Seow, 1998) Este mecanismo es modelado por factores culturales, socio-económicos y conductuales que juegan un poderoso papel en el desarrollo y progresión de esta enfermedad (Reisine \& Douglass, 1998).

A consecuencia de este daño oral, la CTI en los niños puede causar dolor, dificultades funcionales, desordenes de salud general, problemas psicológicos, hospitalizaciones y atenciones de urgencia, lo que conlleva a una menor calidad de vida (Low et al., 1999; Sheller et al., 1997).

En relación a los aspectos epidemiológicos, la CTI constituye un serio problema de salud pública, (Berkowitz, 2003) siendo más prevalente en países en vías de desarrollo como el nuestro y comunidades

\footnotetext{
* Departamento de Odontología Integral, Facultad de Medicina, Universidad de La Frontera, Temuco, Chile.

* Servicio de Salud del Reloncaví, X Región, Chile.

*** Departamento de Salud Pública, Facultad de Medicina, Universidad de la Frontera, Temuco, Chile.
} 
desprotegidas de países desarrollados como son poblaciones de inmigrantes, minorías étnicas o zonas rurales en donde la prevalencia alcanza hasta un $90 \%$ (Miles, 1996).

En Chile existen muy pocos estudios referentes al tema y la prevalencia varía desde un $7,8 \%$ a un $62,3 \%$ (Echeverria et al., 2003; Mariño \& Onetto, 1995). El último reporte ministerial no publicado reveló una prevalencia de un $27 \%$ a los 2 años y de un $48 \%$ a los 4 años (MINSAL, 2007).

El propósito de este estudio fue determinar la prevalencia y severidad de CTI y sus factores relacionados, en niños de 2 y 4 años de edad atendidos en el Hospital de Calbuco, con el fin de tomar medidas atingentes a la realidad local.

\section{MATERIAL Y MÉTODO}

Población. Se realizó un estudio de corte transversal en el Hospital de Calbuco, perteneciente al Servicio Salud del Reloncaví, $X$ región, Chile. Calbuco está localizado a $56 \mathrm{~km}$ al Suroeste de Puerto Montt, capital de la región. Es un archipíelago formado por 14 islas y está unido al continente por un pedraplén. Su población es mayoritariamente de nivel socioeconómico mediobajo.

La población total de la comuna de Calbuco en niños de 2 y 4 años es de 1094, (573 de sexo masculino y 521 de sexo femenino), distribuyéndose en 451 del área urbana (237 = sexo masculino; 214 = sexo femenino), y 643 del área rural (336 = sexo masculino; 307= sexo femenino). De esta población de referencia el $72 \%$ (788 niños) son beneficiarios de los establecimientos públicos de salud.

La selección de la muestra fue determinada por método no probabilístico, de casos consecutivos. Se examinaron todos los niños entre 2 y 4 años que asistieron al programa Ministerial de Control Odontológico de Niño Sano (Controles odontológicos obligatorios), entre enero y diciembre 2008. Se excluyeron los niños con discapacidad, enfermedad sistémica de base y/o atención odontológica previa. Finalmente, la muestra quedó constituida por 301 niños que corresponden al $38 \%$ de los niños beneficiarios del sistema público de salud.

Examen Clínico. Los niños fueron examinados por un solo investigador, calibrado previamente por prueba pi- loto y con un coeficiente de correlación intraclásica de un $98 \%$. Los exámenes fueron realizados en dependencias del Servicio Dental del Hospital de Calbuco, bajo luz artificial e inspección visual de la cavidad oral. La presencia de caries se estableció en base a los criterios propuestos por la OMS en el Oral Health Survey Basic Methods, para estudios epidemiológicos; considerando a la caries incipiente dentro del componente caries. Estas fueron identificadas a través de la presencia de un área blanco-opaca en el tercio gingival de la corona. Para facilitar la detección se realizó una profilaxis previa con cepillo profiláctico, cepillo dental o gasa de acuerdo a la edad o grado de cooperación.

La severidad de CTI fue determinada de acuerdo a los parámetros establecidos por Ismail et al. 1999, (Ismail \& Sohn, 1999).

Se utilizó una ficha clínica diseñada para los objetivos de este estudio, que incluyó antecedentes registrados por el examinador, antecedentes médicos e información otorgada por la madre. Los antecedentes de la ficha clínica fueron validados en la prueba piloto a través de una validación de contenidos.

Descripción de las variables. La ficha clínica registró datos demográficos, alimenticios y de higiene oral. Los pacientes fueron registrados de acuerdo al sector del cual provenían en rural y urbano. La previsión se determinó según el tipo de seguro de salud estatal que poseen, que están en directa relación al nivel socio-económico del grupo familiar. El estado nutricional se registró de acuerdo a las referencias OMS para la evaluación antropométrica adaptadas por el Ministerio de Salud de Chile.

Hábitos de alimentación: se recolectó información acerca del tiempo de lactancia materna (se consideró lactancia materna prolongada a todos los niños que amamantaron después de 1 año), uso biberón actual y uso biberón nocturno. Se realizó una encuesta de dieta para determinar el valor potencial cariogénico (bajo-moderado-alto).

Hábitos de higiene oral: se registro la presencia de higiene oral, frecuencia de cepillado y el índice de Higiene Oral Simplificado de Greene y Vermillion (IHOS)

Plan de análisis. El análisis de los datos fue realizado por medio del programa estadístico stata 10. Las pruebas estadísticas realizadas fueron test exacto de Fisher, considerándose un nivel de significación de un 0,05 y un análisis multivariado con un nivel de confianza de un $95 \%$. 


\section{RESULTADOS}

Descripción de la muestra. La muestra está constituida por 301 niños (Tabla I) que corresponde al 38\% del total de niños beneficiarios del Hospital de Calbuco. Se distribuyeron en 50,8\% (153/301) de sexo masculino, 51,5\% (155/301) niños de 2 años y 48,5\% (146/ $301)$ de 4 años. Un $27,6 \%$ (83/301) pertenece a zonas rurales y un 63\% (189/301) corresponde al nivel A y B del Fondo Nacional de Salud (FONASA). La prevalencia de CTI es de $70 \%$ (211/301), y la prevalencia de CTI-S es de 52\% (156/301), con una mediana para el índice ceod igual a 3. El 28\% (44/155) de los niños de 2 años presentaban lesiones incipientes. (Tabla II)

Tabla I. Descripción de la muestra.

\begin{tabular}{|c|c|c|}
\hline Característica & $\begin{array}{c}\text { Frecuencia } \\
n=301\end{array}$ & $\%$ \\
\hline Sexo masculino & 153 & 50,8 \\
\hline Edad 2 años & 155 & 51,5 \\
\hline Residencia rural & 83 & 27,6 \\
\hline Previsión: FONASA A - B & 189 & 63 \\
\hline $\begin{array}{l}\text { Previsión FONASA C - D - } \\
\text { Isapre }\end{array}$ & 112 & 37 \\
\hline
\end{tabular}

Análisis por objetivos. La asociación entre las variables de control y de exposición con CTI (Tablas III - IV), fue estudiada a través del test exacto de Fisher, para un nivel de significación $a=0,05$. Los resultados del estudio mostraron asociación significativa con CTI para las variables residencia y edad, con un mayor porcentaje de CTI en los rurales y en los niños de 4 años, $81 \%$ y $88 \%$, respectivamente. Similarmente el estado nutricional, lactancia materna prolongada, uso de biberón, uso de biberón nocturno, IHOS se asoció con CTI. Las variables valor potencial cariogénico, aseo bucal, frecuencia de cepillado y cepillado antes de dormir no muestran asociación significativa.

Dada la asociación de las variables de control edad y ruralidad con CTI, fue preciso estudiar la asociación entre las variables de exposición y CTI, ajustándolas a las variables de control mediante un modelo multivariado.

Análisis Multivariado. Al realizar el análisis estratificado, se descubrió que la edad es una importante variable de interacción, por lo cual se decide construir un modelo para cada grupo de edad. El modelo estadístico a utilizar fue el de regresión logística. Dado que este estudio es de corte transversal, el OR como medida de intensidad y dirección de la asociación, se interpretará como OR de la enfermedad.

Modelo en niños de 2 años. Se eliminaron interacciones en forma manual por su significado clínico y mediante el procedimiento de comparación de los estimadores de verosimilitud (Irtest). El modelo final en este grupo de edad no contiene interacciones, siendo significativas las variables sobrepeso/obeso, lactancia materna prolongada, IHOS y residencia rural, con un OR mayor de 1, es decir, son factores de riesgo para CTI. En el caso de la variable de exposición uso de biberón nocturno, el OR

Tabla II. Prevalencia de Caries Temprana de la Infancia.

\begin{tabular}{lccc}
\hline & Total $(\mathbf{n}=\mathbf{3 0 1})$ & $\mathbf{2}$ años $(\mathbf{n}=\mathbf{1 5 5})$ & $\mathbf{4}$ años $(\mathbf{n}=\mathbf{1 4 6})$ \\
\hline Caries temprana de la infancia $(\mathbf{n}(\%))$ & $211(70)$ & $82(53)$ & $129(88)$ \\
Caries temprana de la infancia severa (n (\%)) & $156(52)$ & $66(43)$ & $90(62)$ \\
Caries incipiente ( $\mathbf{n}(\%))$ & $101(34)$ & $44(28)$ & $57(39)$ \\
Lesiones cavitadas (n (\%)) & $185(62)$ & $71(46)$ & $114(78)$ \\
\hline
\end{tabular}

Tabla III. Asociación entre variables de control y CTI.

\begin{tabular}{llcccc}
\hline & Variable & n & CTI & $\%$ & p \\
\hline Residencia & Rural & 83 & 67 & 81 & $0,016^{*}$ \\
\multirow{3}{*}{ Edad } & Urbano & 218 & 144 & 66 & \\
\multirow{2}{*}{ Sexo } & 2 años & 155 & 82 & 53 & $<0,001^{*}$ \\
\multirow{4}{*}{ Nivel Previsión Salud } & 146 & 129 & 88 & \\
& 4 años & 148 & 100 & 68 & 0,379 \\
& Femenino & 153 & 111 & 73 & \\
& Masculino & 111 & 74 & 67 & 0,362 \\
& A-B & 189 & 136 & 72 & \\
\hline
\end{tabular}

*Asociación estadísticamente significativa (test exacto de Fisher). 
fue de 0.378 , es decir, correspondería a un factor protector, observándose 2.6 casos de CTI entre los que no usan biberón nocturno por cada caso de CTI entre los que usan (Tabla V).
Modelo en niños de 4 años. El modelo final en este grupo de edad no contiene interacciones. Todas las variables de exposición, resultaron ser no estadísticamente significativas. (Tabla VI)

Tabla IV. Asociación entre variables de exposición y CTI.

\begin{tabular}{|c|c|c|c|c|c|}
\hline Variable & & $\mathbf{n}$ & CTI & $\%$ & p \\
\hline \multirow[t]{3}{*}{ Estado nutricional } & Desnutridos - bajo peso & 14 & 12 & 86 & $0,003^{*}$ \\
\hline & Normales & 214 & 138 & 65 & \\
\hline & Sobrepeso-obesos & 73 & 61 & 84 & \\
\hline \multirow[t]{2}{*}{ Uso de biberón } & Ausente & 75 & 67 & 89 & $<0,001$ \\
\hline & Presente & 226 & 144 & 64 & \\
\hline \multirow[t]{2}{*}{ Uso de biberón nocturno } & Ausente & 205 & 158 & 77 & $<0,001^{\star}$ \\
\hline & Presente & 96 & 53 & 55 & \\
\hline \multirow[t]{2}{*}{ Lactancia materna } & No prolongada (<1año) & 159 & 102 & 64 & $0,023^{*}$ \\
\hline & Prolongada (>1año) & 142 & 109 & 77 & \\
\hline \multirow{3}{*}{$\begin{array}{l}\text { Indice de higiene oral } \\
\text { s implificado }\end{array}$} & Bajo & 19 & 4 & 21 & $<0,001^{*}$ \\
\hline & Moderado & 162 & 113 & 70 & \\
\hline & Alto & 119 & 93 & 78 & \\
\hline \multirow{3}{*}{$\begin{array}{l}\text { Valor potencial } \\
\text { c ariogénico }\end{array}$} & Bajo & 20 & 15 & 75 & 0,770 \\
\hline & Moderado & 96 & 65 & 68 & \\
\hline & Alto & 185 & 131 & 71 & \\
\hline \multirow{2}{*}{$\begin{array}{l}\text { Realiza prácticas de } \\
\text { higiene oral }\end{array}$} & No & 276 & 196 & 71 & 0,260 \\
\hline & $\mathrm{Si}$ & 25 & 15 & 60 & \\
\hline \multirow[t]{4}{*}{ Frecuencia de cepillado } & Nunca & 24 & 14 & 58 & 0,421 \\
\hline & 1 vez al día & 36 & 28 & 78 & \\
\hline & 2 a 3 veces al día & 195 & 135 & 69 & \\
\hline & Más de 3 veces al día & 45 & 33 & 73 & \\
\hline \multirow[t]{2}{*}{ Cepillado antes de dormir } & No & 197 & 135 & 68 & 0,547 \\
\hline & Si & 103 & 76 & 74 & \\
\hline
\end{tabular}

*Asociación estadísticamente significativa (test exacto de Fisher).

Tabla V. Modelo de regresión logística en niños de 2 años.

\begin{tabular}{lccc}
\hline Variables & Odds Ratio & $\mathbf{9 5 \%} \mathbf{~ C l}$ & $\mathbf{p}$ \\
\hline Estado nutricional (sobrepeso-obeso) & 3,2 & $0,99-10,5$ & 0,051 \\
Lactancia materna prolongada & 2,9 & $1,4-6,0$ & 0,004 \\
Sin biberón nocturno & $1 / 0.378=2.6$ & $1,2-5,7$ & 0,012 \\
Índice de higiene oral simplificado & 5.3 & $1,6-18,3$ & 0,008 \\
Residencia rural & 2.3 & $1,03-5,2$ & 0,043 \\
\hline
\end{tabular}

Tabla VI. Modelo de regresión logística en niños de 4 años.

\begin{tabular}{lcc}
\hline Variables & Odds Ratio & $\mathbf{p}$ \\
\hline Estado nutricional (sobrepeso-obeso) & 0,9 & 0,830 \\
Lactancia materna prolongada & 1,4 & 0,561 \\
Uso de biberón nocturno & 0,5 & 0,313 \\
Índice de higiene oral simplificado & 0,6 & 0,369 \\
Residencia rural & 2,7 & 0,209 \\
\hline
\end{tabular}




\section{DISCUSIÓN}

La población estudiada presenta características socio-culturales-demográficas particulares que consistentemente en la literatura se asocian con altas prevalencias. Los resultados obtenidos, $70 \%$ de CTI y $52 \%$ de CTI-S concuerdan, entonces, con las prevalencias encontradas en otros estudios epidemiológicos realizados en países en vía de desarrollo y poblaciones desfavorecidas de países desarrollados (Berkowitz, 2003; Ismail, 1998; Milgrom et al., 2000; Reisine \& Psoter, 2001; Warren et al., 2008).

En Chile existen escasas investigaciones referentes al tema (Echeverria et al., 2003; Mariño et al., 1995), con prevalencias que varían entre un $7,8 \%$ y un $62 \%$. Estas discrepancias se deben principalmente a los diferentes criterios diagnósticos utilizados y a que las poblaciones estudiadas presentan características sociales y demográficas diversas, imposibilitando la comparación de nuestros resultados. En el año 2007 el Ministerio de Salud de Chile reportó una prevalencia de $17 \%$ en el grupo de 2 años y $48 \%$ en el grupo de 4 años (MINSAL, 2007), siendo menor a la encontrada en nuestro estudio, de un $53 \%$ en niños de 2 años y en un $88 \%$ en el grupo de 4 años. Esta diferencia se debe a que en nuestro estudio fueron diagnosticadas las lesiones incipientes encontrándose una prevalencia de un $34 \%$ de manchas blancas y un $62 \%$ de lesiones cavitadas. Esto nos lleva a pensar, que muchas veces los estudios de salud sub-diagnostican la enfermedad, contribuyendo a que las medidas preventivas instauradas sean muchas veces ineficientes y tardías y que los tratamientos efectuados se tornen temporales e inútiles.

Los resultados muestran una tendencia en el aumento de la magnitud de la enfermedad a medida que la edad también aumenta. Los niños de dos años tienen una prevalencia de un $53 \%$ de CTI, con un $28 \%$ de lesiones no-cavitadas y un $46 \%$ de lesiones cavitadas. En los niños de cuatro años estas cifras aumentan la prevalencia a un $88 \%$, con $39 \%$ de lesiones no-cavitadas y $78 \%$ de lesiones cavitadas. La alta prevalencia de caries en los niños de dos años, sugiere al igual que lo reportado en otros estudios (Tsai et al., 2006; Thitasomakul et al., 2006), que la enfermedad se ha establecido a edad temprana, progresando e incrementando el daño oral a medida que aumenta la edad y, por lo tanto, debería ser considerado un problema mayor que cuando ocurre en individuos de edad más avanzada.
A igual que en otras investigaciones (Milgrom et al., 2000) no se encontró asociación estadísticamente significativa entre CTI y los factores encuesta de dieta cariogénica, aseo bucal, frecuencia de cepillado y cepillado antes de dormir. Esto se debe a los sesgos de información propios de cualquier encuesta o entrevista y que tiene que ver con la deseabilidad social, es decir, responden lo que creen que es correcto o lo que el profesional desea escuchar, ocultando la información verdadera.

Por otra parte se observó asociación estadísticamente significativa entre CTI y las variables estado nutricional, lactancia materna prolongada, uso de biberón, uso de biberón nocturno e IHOS.

Los niños de dos años mostraron asociaciones estadísticamente significativas, siendo indicadores de riesgo de exposición: sobrepeso y obesidad, lactancia materna prolongada, IHOS y residencia rural. El efecto del uso del biberón nocturno en este estudio fue inesperado ( $O R=0,378$ ), lo que coincide con los reportes de otras investigaciones (Carino et al., 2003; Tsai et al., 2006) en donde también se mostró como un factor protector. Este resultado puede verse influenciado por las limitaciones que tiene el estudio en relación a la información recaudada la cual no incluía importantes antecedentes como acerca de la incorporación de algún suplemento azucarado, de la frecuencia del uso del biberón nocturno o si además presentaban lactancia materna al momento del estudio. Al igual que en el estudio realizado por Litt las madres que reportaban el uso de mamadera nocturna también reportaron un alto consumo de azúcares en el niño (Litt et al., 1995).

La asociación entre CTI y lactancia materna a los 2 años (OR de 2,9) es coincidente con los reportes de casos que sugieren que la prolongada y excesiva lactancia materna está asociada con caries rampante en los niños (Seow, 1998) y que la CTI ha sido relacionada a la lactancia materna prolongada más allá del año de vida (Horowitz, 1998). En contra posición, Ribeiro (Ribeiro \& Ribeiro, 2004) presenta una serie de argumentos que refutan la asociación entre lactancia materna y CTI dentro de los cuales están el hecho de que culturas que mantienen hábitos de alimentación ancestrales, sin incorporar hábitos de alimentación actuales (sacarosa) tienen baja prevalencia de caries; otro de los argumentos es que mientras el hábito de lactancia materna ha disminuido y además se extingue con la edad, la prevalencia de CTI ha sido constante y aumenta con la edad. Lo que está claro es que la relación entre caries y lactancia materna es compleja y que 
hay muchas variables biológicas tales como infección de streptococcus mutans, hipoplasia de esmalte, consumo de azúcar y variables como nivel educacional de la madre y/o estado socioeconómico que pueden influir y afectar la salud oral.

Se observó que el factor de sobrepeso y obesidad es un indicador de exposición $(\mathrm{OR}=3,2)$. Esta asociación según Duperon, se debe a que en algunas culturas el tener un niño gordo es sinónimo de un niño saludable o de bebes tranquilos (Duperon, 1995). Por otro lado, algunas madres o cuidadoras dan a los niños chupetes untados en soluciones azucaradas o miel, estas costumbres aún se mantienen fuertemente en nuestra población.

Con respecto al IHOS como indicador de riesgo en niños de 2 años $(O R=5,3)$, es un valor importante destacar, porque esta medición corresponde a un registro realizado por el clínico, siendo más objetiva, y no sólo a una información reportada por la madre y/o el tutor. Horowitz describe que la mayoría de los niños con placa dental visible a los años 1 y 2 años de edad han desarrollado caries a la edad de 3 años con respecto a los niños sin placa dental visible en este mismo rango de edad (Horowitz, 1998).

La residencia rural mostró ser también un factor de exposición, con un OR de 2,3. Este resultado debe analizarse con cautela, porque la población rural incluida en el estudio fue menor que la población urba- na. El Hospital de Calbuco otorga atención odontológica a toda la población urbana y sólo a la población rural que no es cubierta por los otros centros de salud públicos de la Comuna. Los reportes (Reisine \& Psoter, 2001; Warren et al., 2008) indican que los variados niveles de urbanización presentan diferentes niveles de caries, donde los más altos niveles de caries fueron asociados a áreas de baja urbanización. Sin embargo, son necesarios estudios adicionales para determinar hasta qué punto las características demográficas son indicadores de riesgo de caries en poblaciones cuya prevalencia es alta.

Al analizar los resultados del modelo de regresión logística para los niños de 4 años, se observó que las variables estudiadas no tienen asociación estadística significativa. Estos datos coinciden con Tinanoff, que señala que aún no ha sido establecido un único modelo de riesgo para la CTI (Tinanoff et al., 1998). Además, a pesar de que estas variables han sido citadas como clásicos factores de riesgo, la literatura científica ofrece resultados conflictivos del impacto de estos factores sobre la enfermedad (Harris et al., 2004).

Finalmente, este estudio proporciona evidencia importante al otorgar indicadores epidemiológicos significativos para la población que es cubierta por el Hospital de Calbuco. Sin embargo, es necesario realizar estudios adicionales a los indicadores analizados para identificar los factores de riesgo que interfieren complejamente en la prevalencia CTI de esta población.

ZAROR, S. C.; PINEDA, T. P. \& ORELLANA, C. J. J. Prevalence of early childhood caries and associated factors in 2 and 4 year-old Chilean children. Int. J. Odontostomat., 5(2):1171-177, 2011.

ABSTRACT: A cross-sectional study was performed during 2008, with a sample of 301 two and four year-old children attending their first dental appointment in the Calbuco Hospital. The aim of the study was to determine the prevalence of early childhood caries (ECC), severe early childhood caries (S-ECC) and their associated factors. The children's dental examination and caries diagnosis were carried out using standardized methods (World Health Organization). White spot lesion was recorded as caries. The relationship of socio-demographic factors, dietary habits and oral hygiene was also investigated. The data was analyzed using Fisher's exact test and logistic regression models. The prevalence of ECC, including white spot lesions was $70 \%$ with a severity of $52 \%$. A significant statistical association was found between ECC and variables such as age, rurality, nutritional status, bottle-feeding, bottle-feeding at bedtime and simplified oral hygiene index $(\mathrm{OHI}-\mathrm{S})(\mathrm{p}<0.05)$. The multivariate study determined that the presence of bottle-feeding at bed time is a protective factor in 2 year-old children $(\mathrm{OR}=2.6)$. The high prevalence of ECC highlighted the need to incorporate educational and preventive programs during the first year of life.

KEY WORDS: early childhood caries, prevalence, epidemiology, risk factors.

\section{REFERENCIAS BIBLIOGRÁFICAS}

Berkowitz, R. J. Causes, treatment and prevention of early childhood caries: a microbiologic perspective. J. Can. Dent. Assoc., 69:304-7. 2003.
Cariño, K. M.; Shinada, K. \& Kawaguchi, Y. Early childhood caries in Northern Philippines. Community Dent. Oral Epidemiol., 31:81-9, 2003. 
Duperon, D. F. Early childhood caries: a continuing dilemma. J. Calif. Dent. Assoc., 23:15-2, 1995.

Echeverria, S.; Soto, D. \& Zillmann, G: Prevalencia de caries de la Lactancia en niños de 2 a 4 años de la región Metropolitana. Diagnóstico actualizado. $R e-$ vista Dental de Chile, 94:14-8, 2003.

Harris, R.; Nicoll, A. D.; Adair, P. M. \& Pine, C. M. Risk factors for dental caries in young children: a systematic review of the literature. Community Dent. Health, 21:71-85, 2004.

Horowitz, H. S. Research issues in early childhood caries. Community Dent. Oral Epidemiol., 26:6781, 1998.

Ismail, A. I. Prevention of early childhood caries. Community Dent. Oral Epidemiol., 26:49-61, 1998.

Ismail, A. I. \& Sohn, W. A systematic review of clinical diagnostic criteria of early childhood caries. J. Public. Health Dent., 59:171-91, 1999.

Litt, M. D.; Reisine, S. \& Tinanoff, N. Multidimensional causal model of dental caries development in lowincome preschool children. Public Health Rep., 110:607-17, 1995.

Low, W.; Tan, S. \& Schwartz, S. The effect of severe caries on the quality of life in young children. Pediatr. Dent., 21:325-6, 1999.

Mariño, R. J. \& Onetto, J. E. Caries experience in urban and rural Chilean 3 year-olds. Community Dent. Oral Epidemiol., 23:60-1, 1995.

Milgrom, P.; Riedy, C. A.; Weinstein, P.; Tanner, A. C.; Manibusan, L. \& Bruss, J. Dental caries and its relationship to bacterial infection, hypoplasia, diet, and oral hygiene in 6 to 36-month-old children. Community Dent. Oral Epidemiol., 28:295-306, 2000.

Milnes, A. R. Description and epidemiology of nursing caries. J. Public Health Dent., 56:38-50, 1996.

MINSAL. Diagnóstico en Salud Bucal de Niños de 2 y 4 años que asisten a la Educación Preescolar. Región Metropolitana. Disponible en: www.minsal.cl, 2007.

Reisine, S. \& Douglass, J. M. Psychosocial and behavioral issues in early childhood caries. Community Dent. Oral Epidemiol., 26:32-44, 1998.

Reisine, S. T. \& Psoter, W. Socioeconomic status and selected behavioral determinants as risk factors for dental caries. J. Dent. Educ., 65:1009-16, 2001.

Ribeiro, N. M. \& Ribeiro, M. A. Breastfeeding and early childhood caries: a critical review. J. Pediatr. (Rio J.), 80:S199-S210, 2004.

Seow, W. K. Biological mechanisms of early childhood caries. Community Dent. Oral Epidemiol., 26:8-27, 1998.

Sheller, B.; Williams, B. J \& Lombardi, S. M. Diagnosis and treatment of dental caries-related emergencies in a children's hospital. Pediatr. Dent., 19:470-5, 1997.

Thitasomakul, S.; Thearmontree, A.; Piwat, S.; Chankanka, O.; Pithpornchaiyakul, W.; Teanpaisan, R. \& Madyusoh, S. A longitudinal study of early childhood caries in 9- to 18-month-old Thai infants. Community Dent. Oral Epidemiol., 34:429-36, 2006.

Tinanoff, N.; Kaste, L. M. \& Corbin, S. B: Early childhood caries: a positive beginning. Community Dent. Oral Epidemiol., 26:117-9, 1998.

Tsai, A. I.; Chen, C. Y.; Li, L. A.; Hsiang, C. L. \& Hsu, K. $\mathrm{H}$. Risk indicators for early childhood caries in Taiwan. Community Dent. Oral Epidemiol., 34:43745, 2006.

Warren, J. J.; Weber-Gasparoni, K.; Marshall, T. A.; Drake, D. R.; Dhkordi-Vakil, F.; Kolker, J. L. \& Dawson, D. V. Factors associated with dental caries experience in 1-year-old children. J. Public Health Dent., 68:70-5, 2008.

Dirección para correspondencia:

Carlos Zaror Sánchez

Manuel Montt \#112, 4 piso.

Dpto. Odontología Integral

of. 420, Facultad de Medicina

Universidad de la Frontera

Temuco - CHILE

Email: czaror@ufro.cl
Recibido : 19-07-2011 Aceptado: 03-08-2011 